Voix et Images

\title{
Un mouvement de prise de parole : les petits éditeurs de poésie des années 50 et 60 au Québec
}

\section{Richard Giguère}

Volume 14, numéro 2 (41), hiver 1989

L’édition littéraire au Québec

URI : https://id.erudit.org/iderudit/200770ar

DOI : https://doi.org/10.7202/200770ar

Aller au sommaire du numéro

Éditeur(s)

Université du Québec à Montréal

ISSN

0318-9201 (imprimé)

1705-933X (numérique)

Découvrir la revue

\section{Citer cet article}

Giguère, R. (1989). Un mouvement de prise de parole : les petits éditeurs de poésie des années 50 et 60 au Québec. Voix et Images, 14(2), 211-224.

https://doi.org/10.7202/200770ar d'utilisation que vous pouvez consulter en ligne. 


\title{
Un mouvement de prise de parole: les petits éditeurs de poésie des années 50 et 60 au Québec
}

\author{
par Richard Giguère, Université de Sherbrooke
}

\section{Remarque préliminaire}

Comme j'ai déjà esquissé un portrait global de «L'éditeur et l'édition de poésie» des années 40 et $50^{1}$, je ne reviendrai pas sur les statistiques de production et les séries littéraires de cette époque. Mon article se veut plutôt une réflexion sur les conditions de fondation et d'existence d'une dizaine de petites maisons d'édition, sur leurs objectifs et leurs réalisations, et sur les changements qu'elles ont fait subir non seulement à notre façon de voir et de publier un livre de poèmes, mais aussi à notre conception de la poésie même. Nous avons réalisé au Groupe de recherche sur l'édition littéraire au Québec une série d'interviews avec des éditeurs des années 50 et 60 (Roland Giguère d'Erta, André Goulet d'Orphée, Claude Marceau de Nocturne, André Major d'Atys, le père Jean-Louis Brouillé de Cascade, Diane Pelletier-Spiecker et Micheline Sainte-Marie de Quartz, François et Marcel Hébert des Herbes rouges), et même avec quelques-uns des décennies 30 et 40 (Clément Marchand du Bien public, Gilles Hénault des Cahiers de la file indienne, et Lucien Parizeau $)^{2}$. J'ai relu les transcriptions de ces interviews et j'ai pu puiser dans une documentation de première main (catalogues d'éditeur, bibliographies, monographies de maison d'édition) que nous avons accumulée au GRELQ. Je partage par ailleurs quelques intuitions de base avec des chercheurs qui ont publié des travaux sur l'histoire de l'édition littéraire de la première moitié du $\mathrm{XX}^{\mathrm{e}}$ siècle au Québec ${ }^{3}$.

1 Voir mon article dans l'Institution littéraire, sous la direction de Maurice Lemire avec la collaboration de Michel Lord, Québec, IQRC et CRELIQ, 1986, p. 181-189.

2 Ces interviews et cette documentation sont déposés au bureau du Groupe de recherche sur l'édition littéraire au Québec, au Département des lettres et communications de l'Université de Sherbrooke.

3 Voir en particulier les articles de Jacques Michon, «L'édition littéraire au Québec, 1940-1960", dans l'Édition littéraire au Québec de 1940 à 1960, Sherbrooke, Université de Sherbrooke, 1985, p. 1-26 (Cahiers d'études littéraires et culturelles no 9), et «Les revues d'avant-garde au Québec de 1940 à 1979», dans Trajectoires. Littérature et institutions au Québec et en Belgique francophone, sous la direction de Lise Gauvin et Jean-Marie Klinkenberg, Bruxelles/Montréal, Éditions Labor/PUM, p. 117-127, et de Lucie Robert, «Prolégomènes à une étude sur les transformations du marché du livre au Québec (1900-1940)», dans l'Imprimé au 


\section{Situation de l'édition poétique}

Après le boum de la Deuxième Guerre mondiale et de l'immédiat aprèsguerre, l'édition littéraire connaît une période de crise et de déclin de sa production à la fin des années 40 et durant une bonne partie des années 50 . L'édition poétique subit les contrecoups de cette crise car après la Guerre, seules quelques maisons comme Beauchemin et Pilon publient de la poésie: il paraît en moyenne moins de dix titres par année de 1945 à 1950. Il n'est donc pas surprenant que Roland Giguère affirme qu'il ne se faisait pratiquement rien en édition de poésie lorsqu'il lança en 1949 son premier livre, Faire naître, sous le nom des Éditions Erta. En fait, il avait l'impression de fonder sa maison "ex nihilo», ne connaissant à l'époque aucun éditeur qui aurait pu lui servir de modèle 4 . Mais avec la fondation d'Erta, au plus fort de la crise de l'édition littéraire, se crée le premier jalon d'une tradition d'éditeur artisan (et artiste) qui marquera l'édition poétique des années 50 et 60 .

À peu près à la même époque, André Goulet, qui faisait de la peinture tout en fréquentant les cours de l'École des arts graphiques, pressé par un jeune écrivain du nom de Jacques Ferron, commence à imprimer sur une presse manuelle des livres de Ferron d'abord, puis des poètes Jean-Claude Dussault et AndréPierre Boucher. C'est ainsi que naissent les Éditions d'Orphée en 1951. Quant aux Éditions de l'Hexagone, elles sont mises sur pied deux ans plus tard par un groupe d'étudiants ou d'ex-étudiants (Gilles Carle, graphiste, Gaston Miron, cours de sciences sociales, Olivier Marchand, Hélène Pilotte et Louis Portugais, journalistes, Jean-Claude Rinfret, École des beaux-arts) qui, pour la plupart, ont acquis leur première expérience d'édition en publiant le bulletin de l'Ordre de Bon Temps, la Galette (1947-1953) . De même, Éloi de Grandmont des Éditions de Malte et Gilbert Langevin des Éditions Atys s'inscrivent dans cette tradition de jeunes éditeurs: le premier a été étudiant de l'École des beaux-arts de Montréal dans les années 40 alors que le second est encore au Séminaire de philosophie à la fin des années 50. Diane Pelletier-Spiecker et Micheline SainteMarie suivent toutes deux des cours à l'École des beaux-arts de Montréal lorsqu'elles décident de se lancer dans l'édition pour publier leurs premiers recueils de poèmes. Et on pourrait citer combien d'autres exemples semblables, comme Françoise Bujold qui étudie à l'École des arts graphiques et à l'École des beaux-arts de Montréal avant de créer les Éditions Goglin.

\section{La condition et le métier d'éditeur}

Ces petits éditeurs de poésie sont tous des jeunes qui fondent leur maison dans la vingtaine, avec pratiquement pas de capital de départ. La grande majorité d'entre eux sont nés dans des familles où le père est ouvrier, travailleur manuel

Québec. Aspects historiques (18e-20e siècles), sous la direction d'Yvan Lamonde, Québec, IQRC, 1983, p. 225-242 (Culture savante, no 2).

4 Voir mon article «Les Éditions Erta: un surréalisme sans frontières», dans l'Édition littéraire au Québec de 1940 à 1960, p. 142-144.

5 Voir le chapitre qu'Ambroise Lafortune consacre à «L'Ordre de Bon Temps» dans Par les chemins d'Ambroise, Montréal, Leméac, 1983, p. 249-273. 
(Giguère, Goulet, les frères Hébert, Hénault, Langevin, Miron, Fernand Ouellette, Raymond Savard) alors que quelques autres sont de familles petites-bourgeoises ou bourgeoises (Michel Beaulieu des Éditions Estérel, D. Pelletier-Spiecker, M. Sainte-Marie, Gérald Godin des Éditions Parti pris). En général, dans les années 50 , on ne devient pas éditeur de poésie si on n'est pas passé par l'École des arts graphiques (Giguère, Goulet) et l'École des beaux-arts de Montréal (Grandmont, D. Pelletier et Klaus Spiecker, M. Sainte-Marie) et même les deux (F. Bujold), ou si on ne travaille pas déjà dans une maison d'édition commerciale (Miron, vendeur chez Beauchemin, Ouellette, commis-voyageur chez Fides) ${ }^{6}$. De plus en plus, à partir de la fin des années cinquante, un éditeur de poésie donne souvent naissance à un autre éditeur: il y a filiation d'un maître-imprimeur à un apprenti, ou influence, aide et appui. Roland Giguère, lors de son départ pour la France en 1957, vend ainsi tout son matériel d'imprimeur à Pierre Guillaume et Jeannine Leroux qui continueront la pratique de l'édition artisanale et expérimentale d'Erta. Diane Pelletier-Spiecker admet avoir été influencée par les petits livres de Giguère et, en fondant Quartz, demande conseil à André Goulet, puis fait finalement imprimer ses recueils chez Pierre Guillaume et Jeannine Leroux.

En fait, sans l'aide de Giguère, Goulet et Guillaume/Leroux, trois artisansimprimeurs qui travaillent bénévolement ou font des prix d'amis, une partie des éditeurs des années 50 et 60, dont Atys, Quartz, Goglin, les Presses de l'AGÉUM, Estérel, la revue les Herbes rouges au début, n'auraient pas été en mesure de publier les premiers recueils de plusieurs jeunes poètes, dont Michel Beaulieu, Nicole Brossard, Yves-Gabriel Brunet, Françoise Bujold, Pierrre Chatillon, Jean Gauguet, Gilbert Langevin et André Major? ${ }^{7}$. Ces artisans-imprimeurs, qui ont appris leur métier à l'École des arts graphiques, iront achever leur formation par des stages de perfectionnement en France. C'est d'abord Goulet qui partira pour une année, au début de la décennie 50 , puis Giguère y séjournera en tout près de huit ans (1954-1955 et 1957-1963). Celui-ci suivra des cours supérieurs d'arts et de techniques à l'école Estienne de Paris et chez Friedlander, il travaillera aux ateliers Fréquet-Baudier pour la typographie, Desjobert et Mourlot pour la lithographie, Lacoursière et Leblanc pour la taille-douce. Enfin, Gaston Miron fera un séjour de dix-huit mois en 1959-1960 dans le but d'étudier les techniques et les méthodes d'édition (école Estienne). Giguère résume bien l'importance de ces séjours en France pour la nouvelle génération d'éditeurs québécois des années 50 en affirmant que la fréquentation de ces ateliers fut, en quelque sorte, mon université; c'est avec ces artisans que je parachevai mes études. ${ }^{8}$

6 On peut déjà signaler une différence entre les petits éditeurs de poésie des années 50 qui sont des artisans et des artistes et ceux des années 60 qui sont des étudiants en lettres (Michel Beaulieu, Nicole Brossard, André Brochu) et en philosophie (Paul Chamberland, Raoul Duguay, Gilbert Langevin).

7 Ginette Nault, qui a appris le métier de linotypiste et d'imprimeur aux Éditions d'Orphée d'André Goulet, jouera le même rôle de soutien et d'appui pour plusieurs petits éditeurs de poésie des années 70 et 80 .

8 Interview réalisée avec Roland Giguère par Richard Giguère et Hélène Lafrance, 26 octobre 1983, citée par Richard Giguère, «Les Éditions Erta: un surréalisme sans frontières», p. 158. 


\section{Expérimentation et innovation}

La collaboration entre les poètes et les artistes, qui constitue la marque de commerce des Éditions Erta, est reprise par d'autres éditeurs de poésie des années 50. Pendant leur période la plus productive, de 1953 à 1957 - seize livres publiés - , les Éditions Erta expérimentent une variété de techniques, dont la sérigraphie (Jean-Pierre Beaudin) et la lithographie (gravure sur linoléum, sur cuivre, sur zinc) dans l'illustration de leurs livres. Plus que jamais les poètes Hénault, Hæffely, Kœnig, Gauvreau, Bujold, Martino, Horic et les graveurs, les peintres Dumouchel, Anne Kahane, Conrad et Gérard Tremblay, Mousseau, Bellefleur, Beaudin se rencontrent et travaillent ensemble à ce que Denise Marsan appelle l'atelier Erta9. André Goulet affirme en interview que la solidarité entre les artistes était très forte à cette époque. Pour favoriser une complicité entre les poètes et les artistes, il organise des lancements des livres d'Orphée dans les galeries d'art - chez Denyse Delrue, par exemple - et, en 1959 , lorsqu'il a l'idée de lancer la revue Situations, il invite Fernande SaintMartin et le peintre Guido Molinari à faire partie du comité de rédaction. Diane Pelletier raconte que c'est en suivant des cours à l'École des beaux-arts, à la fin des années 50 - c'est d'ailleurs là qu'elle rencontra Klaus Spiecker, artiste immigrant d'origine allemande - , qu'elle apprit à bien connaître le milieu artistique: C'était tellement un petit monde, le milieu artistique de Montréal à cette époque. Il y avait un véritable esprit de village. Cela se résumait peutêtre à cent personnes. ${ }^{10}$ Son amie Micheline Sainte-Marie, qui suivait aussi des cours à l'École des beaux-arts de Montréal et fréquentait l'Échouerie, un lieu de rencontre des peintres, rue Saint-Urbain, va plus loin au sujet de la collaboration entre les poètes et les artistes en affirmant qu'à son avis,

le visuel a devancé le littéraire ici du point de vue de l'invention, de l'avant-garde. Par les arts visuels, plusieurs bouleversements ont eu lieu. [...] les artistes étaient très audacieux. Comme le travail n'était pas verbal, cela passait peut-être un peu mieux dans une première étape. ${ }^{11}$

L'expérimentation et l'innovation chez des éditeurs de poésie comme Erta, Quartz et Goglin, même si les artistes et les poètes travaillent avec des moyens modestes et sur une petite échelle, ne font quand même pas de doute si on les replace dans le contexte éditorial des années 50. De l'édition expérimentale

9 Denise Marsan, «Introduction» au catalogue de l'exposition des Éditions Erta tenue à la Bibliothèque nationale du Québec en mars-avril 1971, Montréal, ministère des Affaires culturelles et Bibliothèque nationale du Québec, 1971, p. 7. Signalons que des poètes comme Hénault, Hæffely, Gauvreau et Bujold ont déjà des rapports étroits avec des artistes avant de publier chez Erta.

10 «Quartz (1958-1960)», interview réalisée avec Diane Pelletier-Spiecker par Sylvie Bergeron, Richard Giguère et Carole Hamelin, le 1er décembre 1984, p. 9-10 (document inédit).

11 «Les Éditions Quartz (1958-1960)», interview réalisée avec Micheline Sainte-Marie par Carole Hamelin, 7 juin 1985, p. 3-4 (document inédit). 
avec des moyens de fortune, déclare Giguère lui-même ${ }^{12}$. Comme à cette époque il n'y avait pas encore de tradition d'édition artisanale et expérimentale en poésie québécoise, Giguère avait toute liberté de faire ce qu'il voulait, d'aller dans la direction qui le tentait. En fait, on peut quasiment dire qu'il n'avait pas le choix, Erta devait innover. Diane Pelletier-Spiecker misait aux Éditions Quartz sur le vers libre, sur l'éclatement du mot et de la phrase, sur le refus des clichés et des stéréotypes et, avec l'aide de Klaus Spiecker et de Pierre Guillaume, sur des livres aux dimensions insolites (format horizontal plutôt que vertical) et aux pages remplies de texte (y compris les quatre pages de couverture) ${ }^{13}$. Quant aux Éditions Goglin de Françoise Bujold, il faut signaler que cette maison a tenté une percée sur le marché du livre d'artiste après le départ de Giguère en France en 1957. Mais cette expérience fut de courte durée et ne connut pas vraiment le succès, car il semble qu'il n'existait pas encore un public lecteur suffisamment connaisseur et/ou intéressé à ce genre de livre produit au Québec à la fin des années $50^{14}$. Cela ne veut pas dire que tous les éditeurs de poésie de cette époque participent à cette politique d'édition expérimentale. L'Hexagone et Atys, par exemple, publient des livres de poèmes d'une conception nettement plus classique. Mais il importe de remarquer que, dans ces deux cas, si on prend le soin d'examiner les circonstances de fondation, les auteurs et les titres publiés, ce sont également les deux maisons qui ont été le moins influencées ou «infiltrées» par des artistes ${ }^{15}$.

\section{Un mouvement de prise de parole}

Un des objectifs des petits éditeurs était de remplir le vide culturel de cette époque, comme le confirment Giguère et Goulet ${ }^{16}$. Pour atteindre ce but, la coutume est de publier ses propres poèmes d'abord, car plusieurs éditeurs sont

12 Roland Giguère, «Une aventure en typographie: des Arts graphiques aux Éditions Erta», Études françaises, vol. 18, no 2, automne 1982, p. 99.

13 Voir le livre de Jean-Paul Martino, Objets de la nuit (Quartz, 1959, 18 p.), où le texte commence sur la page couverture, à la suite du titre, se poursuit sur la deuxième de couverture et se termine sur la troisième et la quatrième de couverture.

14 Cinq ans après son retour de France, en 1968, Giguère recommence à faire paraître des livres sous l'étiquette Erta. Mais conscient du fait que d'autres éditeurs de poésie ont pris la relève pendant son absence, il reprend dans un domaine où lui et Françoise Bujold des Éditions Goglin avaient tenté une percée dans les années 50. C'est-à-dire qu'il publie des livres d'art, des livres d'artiste et des éditions de luxe de poètes de sa génération comme Paul-Marie Lapointe, Robert Marteau et Gaston Miron. Cette fois, il semble qu'il y ait une clientèle de bibliophiles pour ce genre d'édition puisque Erta continue encore de nos jours à publier ses très beaux livres.

15 Rappelons que les Éditions de l'Hexagone sont fondées à Montréal en 1953 par des membres du groupe de l'Ordre de Bon Temps (1946-1953), groupe qui se situe dans le prolongement des mouvements de scoutisme et de guidisme au Québec (voir Ambroise Lafortune, op. cit., p. 115-170, 199-230 et 249-273). Quant aux Éditions Atys, le nom vient du recueil le Sang d'Atys (1940) de François Mauriac, que Langevin admirait beaucoup. Celui-ci, étudiant au Séminaire de philosophie de Montréal et à l'institut Le Guerrier, lance le Mouvement fratemaliste en 1958.

16 André Goulet donne quelques exemples de ce vide culturel ressenti par les écrivains et les artistes au début des années 50: La parution d'un livre, c'était un événement, 
aussi des poètes (Erta, l'Hexagone, Nocturne, Quartz, Goglin, l'Estérel), puis ceux de ses amis - c'est la cas d'Orphée, d'Atys, de Parti pris. On tente aussi de rapprocher l'auteur de son public en produisant des beaux livres à des prix accessibles (1,00 \$ l'exemplaire en moyenne). L'autre objectif important et immédiat est de donner une voix aux poètes. André Major résume bien le climat de la fin des années 50 lorsqu'il rappelle qu'il y avait beaucoup d' effervescence autour de ces petites maisons qui lançaient le mouvement de prise de parole. ${ }^{17}$ Pour la plupart des jeunes poètes qui ne pouvaient pas être publiés et distribués par une maison d'édition commerciale, c'était là le but premier: prendre la parole. À ce sujet, il y avait deux tendances nettement identifiables qui se dégageaient à cette époque et qui ont laissé des traces jusqu'à nos jours. D'un côté, des éditeurs et des poètes «engagés», comme on disait alors, militent dans des groupes ou des partis politiques et ne craignent pas d'afficher leurs couleurs, comme Hénault ou Goulet. De l'autre, quelques éditeurs comme Giguère ou Pelletier-Spiecker, sans être apolitiques, ne voient pas la nécessité d'intégrer explicitement un «message» politique dans leur poésie ou dans leur activité éditoriale. Déjà vers la fin des années 50, et cela deviendra plus évident encore au cours de la décennie 60 , des maisons se donnent comme mission d'édifier une littérature nationale alors que d'autres veulent construire une littérature universelle, pour reprendre l'expression de la directrice des Éditions Quartz ${ }^{18}$.

D'ailleurs, il n'y a qu'à jeter un coup d'œil sur le catalogue des éditeurs pour vérifier la diversité des voix et des tendances de la poésie québécoise de 1950 à 1970 . Il y a une volonté avouée de la part de chaque maison de se distinguer des autres dans le champ éditorial. Erta met l'accent sur la poésie surréaliste, sur ce qui est expérimental et/ou qui se situe dans le prolongement du surréalisme. Elle donne la parole à une nouvelle génération de poètes - Bujold, Gabriel Charpentier, Gauvreau, Giguère, Hénault, Martino -, de quelque origine qu'ils soient: sur les 9 auteurs (21 livres) publiés de 1949 à 1960, Théodore Kœnig est belge, Claude Hæffely est français et Alain Horic, croate. Chez Orphée, Goulet lance une vingtaine de poètes et une trentaine de recueils de 1953 à 1967 . Lui aussi mise sur la nouvelle génération des années 50: Guy Gervais, Gilles Groulx, Michèle Lalonde, Wilfrid Lemoyne et Yves Préfontaine, entre autres. Mais, au contraire d'Erta et avant l'Hexagone, la Barre du jour et les Herbes rouges, il publie aussi des aînés (Maurice Beaulieu, Jean-Charles Harvey) et quelques poètes de la relève des années 60

il fallait souligner la chose. Quand un roman paraissait, on en parlait pendant un mois. La première pièce du TNM, l'Avare [jouée en 1951], a rempli les colonnes des journaux pendant six mois. (Interview avec André Goulet, p. 11)

17 «Atys (1957-1971)», interview avec André Major réalisée par Richard Giguère et André Marquis, 13 mai 1986, p. 23-24 (document inédit).

18 Nous ne cherchions pas à édifier explicitement une poésie nationale, même si c'était très présent et important. [...] Pour nous, il s'agissait de construire une véritable littérature, mais universelle. Nous nous sentions les messagers d'une époque nouvelle ou du Québec «réel». [...] Mais il fallait bien s'appuyer sur notre époque. Notre réalité, c'était l'asphalte, les buildings, le béton. (Interview de D. Pelletier-Spiecker, p. 19-20) 
(Nicole Brossard, Jean-Yves Collette, Louis Geoffroy). L'Hexagone vient vite à représenter, on le sait, le lieu de rencontre, le carrefour des poètes de cette époque. Sur les 25 auteurs (43 recueils) publiés de 1953 à 1970, 15 sont des auteurs maison - Miron, Pilon, Ouellette, van Schendel et Paul-Marie Lapointe comptent parmi les plus connus - et 10 proviennent de différents horizons: ce sont soit des aînés (Lasnier, Grandbois, Hénault), soit des poètes de la même génération (Giguère, Préfontaine, Horic, Godbout), soit des représentants de la relève (Garcia, Péloquin, Brossard). Quant aux Éditions Nocturne, elles publient de 1955 à 1966 des premières œuvres d'auteurs comme Raymond Savard, Roch Carrier, Sylvie Francœur, Monic Nadeau, Georgette Pelletier ou Roger Viau qui souvent ont été refusés ailleurs ${ }^{19}$. Atys, de son côté, fait paraître les recueils des jeunes poètes de la période 1959-1963 (Yves-Gabriel Brunet, Pierre Chatillon, Juan Garcia, Jean Gauguet, Major et Langevin) alors que Quartz et Goglin lancent surtout des femmes (Bujold, Pelletier-Spiecker, Sainte-Marie). Les poètes de Parti pris sont enfin reconnus pour leurs prises de position politiques (Chamberland, Godin) tandis qu'à la même époque, les Presses de l'AGÉUM et l'Estérel publient la relève (Beaulieu, Brochu, Brossard, Duguay, Serge Legagneur et Luc Racine).

En somme, les nouveaux courants voient le jour chez les petits éditeurs qui représentent les forces vives de la poésie québécoise des décennies 50 et 60 . Des critiques et des auteurs d'anthologie comme Gilles Marcotte, Laurent Mailhot et Pierre Nepveu ${ }^{20}$ notent la qualité générale, supérieure à la moyenne, de la poésie publiée à l'Hexagone à cette époque, même par ceux qu'on appelle les "poètes mineurs» 21 de cette maison. On pourrait bien appliquer cette remarque à d'autres éditeurs comme Erta, Orphée, Atys, Quartz, l'Estérel et préciser que cela ne s'explique pas par des raisons de génie spontané, encore moins de génération entière de poètes qui serait plus «inspirée» qu'une autre. Cela tient plutôt au fait que, comme le confirment les interviews que nous avons menées, ce sont les éditeurs-poètes eux-mêmes qui lisaient, acceptaient ou refusaient les manuscrits, puis publiaient les auteurs et les textes retenus sous leur pleine responsabilité. Il n'était pas question de publier un médiocre ou un

19 C'est le cas de Raymond Savard, Roch Carrier et Roger Viau, nous a précisé Claude Marceau en interview, qui ont été refusés par d'autres maisons d'édition avant de s'adresser à l'Union des jeunes écrivains et à Nocturne. La plupart des poètes commençaient par faire paraître quelques textes dans des livres écrits en collaboration, puis, encouragés par la critique et des amis, ils publiaient leurs propres recueils aux Éditions Nocturne. Ce mode de publication des premiers textes dans des numéros collectifs, suivis plus tard par des numéros d'auteur, sera repris à la fin des années 60 et au début des années 70 par les jeunes poètes de la revue les Herbes rouges.

20 Voir l'«Introduction» de Laurent Mailhot et Pierre Nepveu à leur Anthologie de la poésie québécoise des origines à nos jours, Montréal, l'Hexagone, 1986, p. 22-23 (Typo/Poésie), ainsi que l'article «L'Hexagone et compagnie», Liberté, no 120, 1978, p. 11-21 et l'essai de Gilles Marcotte, le Temps des poètes, Montréal, HMH, 1969, p. 15-31, 211-227 et passim.

21 Mentionnons, entre autres, Luc Perrier, Jean-Paul Filion, Claude Foumier, Louise Pouliot, Pierre Trottier, Jacques Godbout, Gilles Constantineau et Gertrude LeMoyne. 
moins bon choix de poèmes d'un auteur parce qu'il fallait atteindre tel nombre de livres produits dans une année pour obtenir de meilleures subventions du Conseil des arts ou du ministère des Affaires culturelles. Cette politique de critères de sélection élevés s'est maintenue tout au long des années 50 et 60 et jusqu'au milieu de la décennie $70^{22}$. Mais cela ne veut pas dire pour autant que la poésie se vendait beaucoup plus ou mieux avant cette date.

\section{Problèmes de vente et de distribution}

Les fondateurs et les fondatrices de maisons d'édition que nous avons rencontrés sont d'accord sur ce point: il n'était pas facile de distribuer et de vendre des livres de poèmes dans les années 50. Si Diane Pelletier-Spiecker affirme que le milieu artistique et littéraire de l'époque se résumait à une centaine de personnes, André Goulet se souvient de son côté que le réseau d'imprimeurs, d'éditeurs commerciaux et de libraires intéressés un tant soit peu à la poésie se limitait à une dizaine de noms. Mis à part les petites maisons qui font l'objet de mon article, seules Beauchemin, Fides et Granger, selon Goulet, publiaient de la poésie. Parmi les libraires, seuls Henri Tranquille, Ducharme (Gérard Malchelosse), Pony et Déom prenaient quelques exemplaires des titres d'Orphée en consigne. Et les deux imprimeries spécialisées dans le livre étaient celles des Éditions Beauchemin et l'imprimerie Saint-Joseph (aujourd'hui l'imprimerie Élite). En ce qui conceme la diffusion du livre de poèmes, il n'y avait aucun distributeur intéressé à s'en occuper à cette époque. Giguère, Goulet, Major, D. Pelletier-Spiecker, Roch Carrier se chargeaient eux-mêmes de faire le tour des quelques librairies qui prenaient cinq ou dix exemplaires de leurs recueils en consigne. Dans un réseau de bibliothèques (publiques et privées) encore peu développé, Goulet raconte que la bibliothèque de l'Université de Montréal retournait les livres qu'il lui envoyait gratuitement, la bibliothèque Saint-Sulpice, elle, les acceptait et lui écrivait un petit mot de remerciement par retour du courrier, mais seule la Bibliothèque juive achetait réellement tous les titres d'Orphée ${ }^{23}$.

Dans ces conditions, comment un petit éditeur devait-il s'y prendre pour assurer une certaine continuité à sa production poétique, surtout si l'on tient compte du fait que les tirages des recueils ne dépassaient guère 500 à 1000 exemplaires en moyenne24? Sauf Orphée et Goglin, tous tentent de garantir cette continuité, de programmer leur édition en créant des collections. Erta met sur pied la «Collection de la Tête armée»25, 6 titres de 1953 à 1957, qui publie

22 Selon Gaston Miron, c'est à partir du milieu des années 70 que les chiffres de vente des livres de poésie se mettent à diminuer au Québec. Le programme des subventions globales aux éditeurs, subventions basées sur le nombre de livres publiés par chaque maison l'année précédente, a été mis en place en 1972 au Conseil des arts du Canada.

23 Interview d'André Goulet, p. 19.

24 Les tirages des livres de poésie varient de 85 exemplaires en moyenne chez Erta (de 1949 à 1960) à 500 chez Orphée ou Quartz., 1000 à l'Hexagone et jusqu'à 1500 parfois chez Beauchemin ou Fides.

25 L'idée de la «Collection de la Tête armée» vient de Claude Hæffely qui par ailleurs a déjà dirigé une petite maison d'édition à Paris, les Éditions Rouge Maille (19461950), avant de venir au Canada. 
les poètes les plus identifiés à la maison (Giguère, Hénault, Kœnig). L'Hexagone lance la collection «Les matinaux» qui, petit à petit, établira sa réputation en publiant de 1954 à 1967 quinze recueils par autant de poètes qui forment le noyau central de la maison (entre autres Perrier, Pilon, Ouellette, Filion, Marchand, van Schendel, Constantineau et P.-M. Lapointe). Quartz avec sa collection «Le refus de la colombe», Atys avec «Silex», Parti pris avec «Paroles», Déom avec «Poésie canadienne» et, plus tard, dans les années 70, les éditions les Herbes rouges, les Écrits des Forges et le Noroît reprendront la même stratégie. Il faut préciser toutefois que cette stratégie, même si elle contribue à fixer l'image de marque d'une maison et, parfois, à faire vendre des livres ${ }^{26}$, connaîtra plus ou moins de succès suivant les éditeurs, les poètes et les époques concernés.

Si les livres de poèmes, tirés à quelques centaines d'exemplaires et mal distribués, ne se vendent pas très bien ${ }^{27}$, la question qui vient logiquement à l'esprit est la suivante: comment les éditeurs réussissent-ils à survivre et dans certains cas à maintenir, voire à augmenter leur rythme de publication? La réponse se trouve dans le mode de financement des maisons. Il faudrait plutôt écrire des modes de financement, car les éditeurs durent inventer toutes sortes de moyens pour financer leur entreprise, surtout avant l'arrivée des subventions gouvernementales. Bien sûr, toutes les maisons fonctionnaient sans but lucratif et la plupart devaient faire appel au compte d'auteur pour s'en sortir. Mais nous nous sommes aperçus, au fil des interviews, que tous les cas sont intéressants et different sensiblement les uns des autres. Atys pratiquait le compte d'auteur classique: Langevin acceptait un manuscrit, le portait chez l'imprimeur et, une fois le livre assemblé, c'est l'auteur qui payait la note et se retrouvait avec les exemplaires sur les bras, obligé de les distribuer lui-même ${ }^{28}$. Orphée avait un mode de fonctionnement particulier. Le poète payait l'encre et le papier, mais Goulet fournissait la main-d'œuvre gratuitement et organisait le lancement. De plus, il conseillait à l'auteur d'écrire au Secrétariat de la Province pour demander l'aide du gouvernement, en n'oubliant pas d'inclure un certain nombre d'exemplaires de son recueil (à 1,00 \$ la copie par exemple); le secrétaire Jean Bruchési répondait par un mot d'encouragement accompagné d'un

26 Je me suis rendu compte, en étudiant les Editions Erta, que les critiques des revues et des journaux écrivaient plus de comptes rendus au sujet des livres de poèmes faisant partie d'une collection connue. Par contre, Roland Giguère affirme que, même si les titres de la «Collection de la Tête armée» se sont mieux vendus que les autres livres d'Erta, il a fallu des années pour écouler les stocks.

27 C'est là la règle générale, si l'on se fie aux interviews que nous avons réalisées avec des éditeurs des années 50 et 60 . Mais il y a des exceptions, et Goulet par exemple insiste sur le fait que les recueils de Wilfrid Lemoine et de Michèle Lalonde se vendaient très bien aux Éditions Orphée. Presque chaque maison, il semble, a quelques bons vendeurs.

28 André Major nous a raconté sa première expérience de publication aux Éditions Atys, alors qu'il s'est retrouvé avec des centaines d'exemplaires de son recueil sur les bras et une dette importante qu'il a dû̀ rembourser à son père, résultat d'un emprunt pour payer les frais d'imprimerie. 
chèque établi au nom du poète 29 . Nocturne a trouvé une formule originale qui se situe à mi-chemin entre le compte d'auteur et l'auto-édition. Le poète défrayait le coût d'impression de son livre alors que les Éditions Nocturne et l'Union des jeunes écrivains se chargeaient de la mise en pages, du montage et de la correction d'épreuves. C'est également l'Union et Nocturne qui guidaient l'auteur dans ses relations avec l'imprimeur et l'aidaient en principe à distribuer son livre ${ }^{30}$. Diane Pelletier-Spiecker finance les Éditions Quartz en partie par le compte d'auteur, en partie par la vente d'une fraction des tirages au Secrétariat de la Province et en partie par les sommes amassées grâce à une liste de souscripteurs (pré-vente qui aide à payer les frais d'imprimerie). Cette liste de souscripteurs est devenue une pratique courante des petits éditeurs au Québec depuis les débuts de l'édition. Roland Giguère, enfin, précise au sujet de sa maison deux particularités qui sont uniques à Erta: d'abord, il imprime un livre avant de faire appel à une liste de souscripteurs et surtout, caractéristique rare, sa maison n'a jamais demandé un sou de subvention à l'État.

\section{Revues littéraires et ouvertures sur le monde}

Il existe pour un éditeur un moyen de mousser la vente de ses livres. Il s'agit de créer une revue qui fait la publicité des auteurs maison, publie leurs textes, fait circuler leurs noms et ménage bien sûr un accueil critique favorable à leurs publications. On oublie trop souvent que ce sont des petits éditeurs de poésie qui sont à l'origine des revues littéraires et culturelles les plus dynamiques de la fin des années 50. Il ne fait pas de doute que Situations (1959-1962) et Liberté (fondée en 1959) sont des prolongements des maisons d'édition Orphée et l'Hexagone et que, fondées la même années, elle se retrouvent en compétition au titre de revue porte-parole des intellectucls d'une même génération. Au départ, sur papier, les deux comités de rédaction sont d'égale compétence, car ils font appel à des jeunes représentant la génération montante, c'est-à-dire aussi bien des poètès, des romanciers, des essayistes que des peintres, des journalistes, des enseignants et des étudiants. Le comité de rédaction de Situations est composé de neuf membres - Jacques Archambault, Jean Depocas, Guy Fournier, Jacques Ferron, Michèle Lalonde, Guido Molinari, Yves Préfontaine, Fernande SaintMartin et Maurice Beaulieu, secrétaire de rédaction - dont la très grande majorité, en publiant chez Orphée, demeurent fidèles à la maison. On peut faire la même remarque au sujet des neuf membres fondateurs de Liberté - André Belleau, Gilles Carle, Jean Filiatrault, Jacques Godbout, Gilles Hénault, PaulMarie Lapointe, Fernand Ouellette, Michel van Schendel, Lucien Véronneau réunis autour de Jean-Guy Pilon. Mais bientôt, des caractéristiques de plus en

29 La pratique existe depuis les années 20 et 30 . Simone Routier et Alfred DesRochers, entre autres, ont sollicité et obtenu l'aide financière du Secrétariat de la Province pour payer l'impression de leurs recueils l'Immortel Adolescent (1928) et À l'ombre de l'Orford (1929), de même que Clément Marchand pour plusieurs livres parus aux Éditions du Bien public.

30 C'est l'originalité de cette formule qui a attiré de nombreux jeunes auteurs non seulement de Montréal, mais de différentes régions de la province et même de l'extérieur du Québec à devenir membres de l'Union des jeunes écrivains. 
plus marquées se transforment en traits distinctifs de chacune des deux revues et font pencher la balance du côté du groupe de l'Hexagone. Pratiquement dès le départ, Situations est politiquement plus engagée que Liberté qui, sous la direction de Pilon, évince les éléments radicaux (Hénault, van Schendel, puis Hubert Aquin) de son comité de rédaction. À la longue, la crédibilité individuelle et institutionnelle (Radio-Canada, Office national du film) du directeur et des rédacteurs de Liberté dépasse celle du groupe des Éditions d'Orphée. Enfin, la permanence de Liberté est assurée par un Jean-Guy Pilon qui, réalisateur à Radio-Canada, se trouve dans une position de pouvoir et d'influence indéniablement supérieure à celle de l'imprimeur André Goulet. Celui-ci, d'un numéro à l'autre quasiment, est continuellement à la recherche d'un secrétaire de rédaction permanent qui donnerait une certaine stabilité à son équipe et à sa revue ${ }^{31}$.

Orphée et l'Hexagone ne sont pas les seules maisons qui donnent plus d'ampleur à leur travail d'édition en fondant une revue littéraire et culturelle. D'autres éditeurs mettent sur pied ou contribuent à mettre sur pied des revues qui accroissent le champ d'activités des poètes et le rayonnement de leurs œuvres, qui les amènent aussi à prendre position face qux problèmes sociopolitiques et à s'ouvrir au monde extérieur. En simplifiant un peu vite deux décennies d'histoire, on a eu souvent tendance au Québec à identifier les années 50 et la première moitié des années 60 à une période de nationalisme replié sur lui-même alors que dans les faits, l'action des éditeurs de poésie et des revues littéraires et culturelles prouve exactement le contraire. Erta, par exemple, je l'ai déjà signalé, est dirigée par un Roland Giguère qui s'associe au mouvement surréaliste français et qui publie des poètes d'origine étrangère. Giguère et son collaborateur Claude Hæffely, lors de leur départ pour Paris en 1957, projetaient même de lancer là-bas une collection de poètes francophones hors Québec. Ce projet ne verra pas le jour et sera remplacé par une petite revue de création animée par Hæffely et qui fonctionne par correspondance, le Périscope («Cahiers des poëtes d'expression française», 1958-1960). En plus de son directeur, la revue a des éditeurs correspondants au Canada, aux États-Unis, en Suisse, en Belgique et fait parâtre en huit livraisons trimestrielles, 15 poètes québécois et 30 poètes francophones - belges, suisses et français surtout ${ }^{32}$. De son côté, Diane Pelletier-Spiecker a collaboré à la fondation de la revue

31 Maurice Beaulieu, Jacques Ferron, Robert Millet, Louis Martin et Patrick Straram se succèdent tour à tour à la barre de Situations qui publie 16 numéros en trois ans et demi d'existence. Liberté en est à la fin de 1988 à son $180^{\circ}$ numéro en trente ans.

32 Les quinze poètes québécois publiés dans le Périscope, revue tirée à 350-400 exemplaires du printemps 1958 à l'été 1960, sont surtout ceux d'Erta (Giguère, Hæffely, Hénault) et de l'Hexagone (Lapointe, Marchand, Miron, Ouellette, Pilon, Portugais, van Schendel), mais aussi des femmes (M.-C. Blais, F. Bujold, M. Sainte-Marie) et quelques jeunes moins connus (André Contant, Guy Gervais, Alma de Chantal). Dans le dernier numéro paru, Hæffely lance l'idée de poursuivre l'aventure du Périscope en publiant une nouvelle série de numéros consacrés à un seul ou à quelques auteurs, tout comme les Herbes rouges le feront pour une nouvelle génération de poètes québécois à la fin des années 60 . Voir le Périscope, textes et illustrations réunis par Claude Hæffely, huit numéros de 16 pages reliés 
Exchange avec des poètes canadiens-anglais alors que Gilbert Langevin d'Atys et Claude Marceau de Nocturne se proposaient aussi de fonder des périodiques ${ }^{33}$.

L'ouverture sur le monde est manifeste chez tous ces éditeurs si l'on examine de près leur politique éditoriale et leur catalogue. Ce qui intéresse Erta, c'est le rayonnement de la poésie surréaliste de langue française, au Québec, en France et en Belgique en particulier. André Goulet publie aux Éditions d'Orphée le poète juif montréalais Irving Layton (A Laughter in the Mind) dès 1958, imprime des revues en anglais pour les universités Sir George Williams et McGill, et fait même la composition typographique des premiers numéros de la revue du poète ontarien James Reaney, Alphabet (London, Ontario, 19 numéros, 19601971). Plus tard, dans les années 70 et 80 , il publiera en espagnol des recueils de poètes exilés du Chili, de l'Argentine, du Guatemala et du Salvador. Les Éditions de l'Hexagone d'abord, puis Liberté par la suite organisent les Rencontres de poètes annuelles de 1957, 1958 et 1959, qui deviennent des Rencontres d'écrivains canadiens en 1960 et 1961 pour finalement se transformer, à la fin des années 60 , en Rencontres québécoises internationales des écrivains. On voit très bien, dans ces deux cas, l'ouverture progressive du Québec sur le Canada, sur l'Amérique et sur le monde. On connaît aussi l'intérêt de Parti pris pour la problématique de la décolonisation en Afrique, telle que théorisée par Jacques Berque, Albert Memmi et Frantz Fanon. Quant à Diane Pelletier-Spiecker et à Micheline Sainte-Marie, elles sont toutes deux plus attirées dans les années 50 par New York, le jazz américain, le musée Guggenheim et Rockefeller Centre que par Paris et la culture des «vieux pays». L'une enseignera à Vancouver et à Ottawa et est aujourd'hui responsable du Bureau de traduction du Musée des beaux-arts de Montréal, l'autre aide à créer une revue bilingue, publie un poète torontois et un américain, et participe avec Irging Layton à une tournée de récitals de poésie dans l'Ouest canadien financée par le Conseil des arts du Canada.

\section{Une nouvelle tradition}

En fin de compte, en provoquant une remise en question du recueil de poèmes classique et sa façon de le mettre en livre, en bâtissant des réseaux de collaboration entre les poètes et les artistes et en sortant de son isolement la poésie écrite en français en Amérique du Nord, les petites maisons des années 50 et 60 créent une nouvelle tradition de l'édition poétique au Québec. Tout cela n'arrive pas du jour au lendemain, bien sûr, mais se produit tout de même dans une période relativement courte de deux décennies et dans le contexte des changements rapides des années 60 . Il ne faut pas oublier en effet que c'est autour de 1960 - un peu avant ou un peu après cette date - que le Conseil des arts du Canada (1957) et le ministère des Affaires culturelles du Québec (1961)

par les Presses Elite, en décembre 1978, pour le plaisir de l'Hexagone à l'occasion du vingt-cinquième anniversaire de sa fondation.

33 Les Cahiers fraternalistes de Gilbert Langevin, qui ont publié un seul numéro en 1964 (Silex no 5, publication bimestrielle du centre de recherches fraternalistes), affirmaient avoir un représentant distributeur des Éditions Atys en Europe: François Hertel, 65 rue d'Amsterdam, Paris 8. 
sont créés, que des Délégations générales du Québec sont ouvertes à New York et à Paris (1961), que des accords sont signés entre la France et le Québec en matière d'éducation et de culture (1965). À la faveur de la Révolution tranquille, on assiste à une éclosion des relations internationales du Québec avec la France en particulier, mais non pas exclusivement. Ces événements ont eu de nombreuses retombées dans le domaine de la culture et de la littérature. Mon intention n'est pas de les énumérer toutes, mais d'évoquer en concluant celle qui a eu le plus d'impact sur l'édition poétique de 1960 à nos jours.

Au cours des décennies 40 et 50 , plusieurs artistes et écrivains ont fait des séjours plus ou moins prolongés en France, surtout à Paris ${ }^{34}$. Certains même comme Paul-Émile Borduas, Jean-Paul Riopelle, Fernand Leduc, François Hertel, Jean-René Major s'étaient exilés et ne revenaient plus au Québec que sporadiquement. Après la Révolution tranquille, le mouvement d'exil ou de séjours en France diminue sensiblement et même se réoriente en partie vers New York. Ou alors les séjours d'écrivains et d'artistes à l'étranger prennent place dans le cadre d'échanges culturels signés par les gouvernements québécois et français, américain, italien, allemand, etc. La nouvelle génération d'artistes et surtout d'écrivains et de poètes qui commencent à émerger vers la fin des années 60 ne sent plus avec la même urgence la nécessité de séjourner longuement à Paris ou à New York, encore moins de s'exiler à vie. Cela a des répercussions sur l'édition littéraire puisque dorénavant des écrivains et des poètes décident de s'enraciner, d'écrire et de publier ici.

La nature et le statut de l'éditeur de poésie s'en trouvent changés. Alors que dans les années 40 , des maisons d'édition commerciale faisaient une toute petite place à la poésie, le plus souvent à compte d'auteur comme chez Beauchemin, Fides ou Granger, dans les années 50, ce sont des poètes eux-mêmes, des artistes et des artisans du livre qui prennent en main l'édition de leurs livres en fondant leurs propres maisons d'édition tandis que dans les années 60 et 70 , ce sont des étudiants qui aspirent à devenir poètes qui fondent les revues et les maisons d'édition de poésie. Les comités de rédaction de Parti pris, de la Barre du jour, de Passe-partout, de Quoi et des Herbes rouges sont surtout composés d'étudiants en philosophie ( $P$. Chamberland, R. Duguay, G. Langevin) et en lettres (M. Beaulieu, C. Beausoleil, N. Brossard, J.-Y. Collette, N. de Bellefeuille, M. Gay, G. Saint-Pierre, R. Soublière. F. Théoret). Ces changements dans l'édition poétique des années 40 et 50 aux années 60 et 70 auront des conséquences non seulement sur le type de poètes et les séries publiés, mais aussi sur l'évolution formelle de l'écriture poétique. Chez Roland Giguère par exemple, le poème est construit comme un ensemble, écrit d'un

34 Le phénomène n'est pas nouveau à cette époque. Des écrivains et des artistes québécois font des séjours plus ou moins longs en France depuis les premières décennies du 20c siècle (Paul Morin, René Chopin, Guy Delahaye, Marcel Dugas, Robert de Roquebrune), et même depuis le XIX'e siècle dans certains cas (l'abbé Casgrain, Octave Crémazie). Mais l'habitude de ces séjours, réservée aux fils et aux filles de familles bourgeoises aisées durant la première moitié du $\mathrm{XX}^{\mathrm{e}}$ siècle, s'intensifie après la Deuxième Guerre mondiale. 
seul jet et publié avec très peu de retouches si l'on compare la version finale avec le manuscrit original. Et on pourrait faire les mêmes remarques pour d'autres poètes d'Erta, de Quartz ou de Goglin qui ont reçu une formation en arts visuels. Pour certains poètes qui pratiquent la profession de journaliste comme Godbout, Godin, Hénault, Lapointe, Major, van Schendel, des préoccupations socio-politiques apparaissent clairement dans leurs recueils des années 50 et 60 . Chez les Beaulieu, Brossard, Collette, Gay et autres qui commencent à publier au milieu des années 60 et dans les années 70 , étudiants en lettres nourris de structuralisme, de sémiotique et rompus à l'analyse systématique des textes, le travail formel du poème est de première importance.

Paradoxalement, même si la période qui va des années 40 et 50 aux années 60 et 70 en est une de nombreux changements, comme nous venons de le souligner, il reste que l'expérience de ces quelques décennies d'édition voit la naissance d'une nouvelle tradition chez les petits éditeurs de poésie. Que ce soient des maisons - l'Hexagone, Orphée - qui fondent des revues - Liberté, Situations - à la fin des années 50, ou des revues - Parti pris, la Barre du jour, les Herbes rouges - qui fondent des maisons dans les années 60 , il reste que des liens étroits continuent d'exister entre les éditeurs et les revues littéraires et culturelles. Et, peu importe si cela s'est produit consciemment ou non, ce sont des éditeurs de la décennie 50 qui ont servi de modèles aux nouveaux éditeurs des décennies 60 et 70 . La tradition des "petits livres bien faits», des livres d'art et des livres d'artiste d'Erta a été poursuivie par le Noroît qui est reconnu pour la présentation matérielle soignée de ses recueils et pour les éditions d'art du Noroît bibliophile. L'imprimeur et éditeur André Goulet a formé et encouragé des jeunes à se lancer dans l'édition eux-mêmes, comme Michel Beaulieu des Presses de l'AGÉUM et de l'Estérel, comme les frères Hébert de la revue les Herbes rouges. Gaston Miron enfin, le grand animateur de la poésie québécoise des trente dernières années, a été le co-directeur des éditions de l'Estérel, a entretenu des rapports étroits avec les rédacteurs de la revue et les directeurs des Éditions Parti pris, a aidé financièrement François et Marcel Hébert à fonder leur propre maison d'édition, et quoi encore 35 ! Malgré les apparences, il y a une profonde continuité dans l'édition poétique québécoise des années 50 aux années $80^{36}$.

35 Seule une interview bien préparée avec Gaston Miron pourrait nous montrer l'importance capitale de son rôle comme animateur et comme éditeur de la littérature québécoise depuis trente ans. Nous attendons toujours qu'il tienne sa promesse maintes fois répétée de nous accorder cette interview.

36 Le parallélisme le plus fascinant et à première vue le plus improbable pourrait être tracé entre les Éditions Nocturne (1955-1966, 31 livres) et les premières années de la revue les Herbes rouges (1968-1974, 21 numéros). Ce parallélismé ne porterait pas sur les auteurs eux-mêmes ni sur les séries poétiques privilégiées par Claude Marceau et Raymond Savard d'une part et par François et Marcel Hébert d'autre part, mais sur le nombre total d'auteurs publiés ( $74 \mathrm{chez}$ Nocturne, 78 aux Herbes rouges), sur le noyau d'auteurs maison (12 chez Nocturne, 9 aux HR), sur le principe de l'alternance des livres ou numéros écrits en collaboration et des livres ou numéros d'auteur (13 livres écrits en collaboration et 17 recueils individuels chez Noctume, 8 collectifs et 13 numéros d'auteur aux HR). Ce parallélisme pourrait même inclure en partie le mode de financement et de fonctionnement des deux éditeurs. 